Revue d'histoire de l'Amérique française

REVUE D.HISTOIRE DE L'AMÉRIQUE FRANÇAISE

\title{
CASTONGUAY, Stéphane et Michèle DAGENAIS, dir., Metropolitan Natures. Environmental Histories of Montreal (Pittsburgh, University of Pittsburgh Press, 2011), 321 p.
}

\section{Harold Bérubé}

Volume 65, numéro 4, printemps 2012

URI : https://id.erudit.org/iderudit/1021051ar

DOI : https://doi.org/10.7202/1021051ar

Aller au sommaire du numéro

Éditeur(s)

Institut d'histoire de l’Amérique française

ISSN

0035-2357 (imprimé)

1492-1383 (numérique)

Découvrir la revue

Citer ce compte rendu

Bérubé, H. (2012). Compte rendu de [CASTONGUAY, Stéphane et Michèle DAGENAIS, dir., Metropolitan Natures. Environmental Histories of Montreal (Pittsburgh, University of Pittsburgh Press, 2011), 321 p.] Revue d'histoire de l'Amérique française, 65(4), 503-505. https://doi.org/10.7202/1021051ar d'utilisation que vous pouvez consulter en ligne.

https://apropos.erudit.org/fr/usagers/politique-dutilisation/ 
CASTONGUAY, Stéphane et Michèle DAGENAIS, dir., Metropolitan Natures. Environmental Histories of Montreal (Pittsburgh, University of Pittsburgh Press, 2011), 321 p.

Tiré d'un atelier qui s'est tenu à Montréal en mars 2009, cet ouvrage rassemble une quinzaine de contributions sur l'histoire de la métropole québécoise. La majorité de ces textes s'inscrivent résolument dans le champ de l'histoire environnementale, alors que d'autres mobilisent le concept d'environnement urbain dans un sens beaucoup plus large. Dans leur introduction, les directeurs, Stéphane Castonguay et Michèle Dagenais, définissent d'ailleurs très généreusement le concept d'environnement et, dans ce qui est pratiquement devenu un passage obligé lorsque vient le moment d'aborder une telle notion, en soulignent la pluralité, la nature construite - une mise au point reprise dans plusieurs des chapitres. De même, s'ils offrent une nécessaire introduction à Montréal - l'archipel et la ville - aux lecteurs qui ne seraient pas familiers avec leur objet de recherche (ce qui risque d'être le cas de la majorité d'entre eux), certains de ces éléments de contexte sont répétés dans plusieurs des chapitres qui suivent. Enfin, ce sont là des défauts bien mineurs et caractéristiques de ce genre d'ouvrage, et ils ne devraient pas porter ombrage à ce qui est clairement une contribution substantielle à l'histoire de Montréal.

Les chapitres sont organisés selon trois grands thèmes. Sous le premier thème, cultures urbaines et représentations, on retrouve le texte plutôt descriptif de Colin Coates sur les représentations coloniales de la ville ainsi que celui de Victoria Dickenson, qui risque de laisser le lecteur perplexe. Elle tâche d'y revivre les expériences sensorielles qu'ont eues certains des premiers Européens à visiter l'île. Si son texte ne manque pas de 
poésie, il tient plus du témoignage personnel que de l'étude historique. Plus intéressants, les textes de Nicolas Kenny et Magda Fahrni traitent de la façon dont les citadins ressentent et vivent l'expérience urbaine à travers leurs sens, leur corps. Le premier le fait en se penchant sur l'impact environnemental de l'industrialisation de la ville, alors que la seconde s'intéresse à l'épidémie d'influenza qui touche la métropole dans les années qui suivent la Première Guerre mondiale. Comme le souligne Kenny, ces expériences sensorielles jouent un rôle central dans la façon dont les citadins conçoivent l'expérience urbaine et s'y situent, mais aussi dans les rapports de pouvoir qui se déploient dans cet environnement (p. 59). C'est aussi vrai des élans d'enthousiasme qu'inspirent la beauté du milieu géophysique et les cheminées d'usine qui s'élancent vers les cieux, que des peurs que suscite la fumée noire que crachent ces cheminées et les conditions dans lesquelles vivent ceux qui sont touchés par l'influenza.

Le second thème, systèmes sociotechniques et infrastructures urbaines, permet de rassembler des textes qui se répondent bien, et en particulier ceux de Dany Fougères, Michèle Dagenais, Susan M. Ross et Christopher G. Boone qui traitent des rapports complexes qu'entretient la ville avec l'eau, qu'il s'agisse de la consommer, de la purifier, de l'évacuer ou d'en contrôler le niveau. Les textes de Fougères et de Dagenais, qui ont consacré d'importantes monographies au sujet, permettent de suivre sur plus de deux siècles le développement des infrastructures liées à la gestion de l'eau sur l'île. Le texte de Ross, extrêmement intéressant, se penche sur ces réservoirs creusés à même le mont Royal au fil des ans et dissimulés dans ce paysage «naturel». Elle y propose notamment une réflexion intéressante sur l'historicité de l'idée de pureté de l'eau. Boone, pour sa part, traite des jeux politiques très complexes qui entourent la gestion des inondations dont sont victimes certaines sections de la ville dans les dernières décennies du XIX ${ }^{\mathrm{e}}$ siècle. Dans les quatre cas, on parle autant de préoccupations environnementales - et en particulier de tout ce qui touche aux questions de santé et de salubrité - que de transformations majeures de l'environnement et du rapport à l'environnement. Ces études permettent également d'observer comment ces transformations dépendent souvent d'une volonté politique et de ressources économiques qui fluctuent énormément dans le temps selon la conjoncture, inondations et épidémies étant des motivateurs dont les effets sont aussi puissants qu'éphémères.

Cela dit, je ne voudrais pas passer sous silence le brillant chapitre de Sherry Olson sur les rues comme éléments de l'environnement urbain. 
Elle montre bien l'influence énorme qu'ont ces espaces publics sur la façon dont se développe le tissu urbain et elle projette habilement sa réflexion à l'aube du XXI ${ }^{\mathrm{e}}$ siècle pour relever les défis auxquels font face les autorités municipales dans l'utilisation durable du réseau routier urbain et des infrastructures qui lui sont liées. Ce sont des thématiques qui surgissent également dans le chapitre que consacre Claire Poitras au développement du réseau autoroutier montréalais, développement étroitement lié à la tenue de l'Expo 67. Elle s'intéresse aux choix qui ont été faits dans ce contexte et aux coûts environnementaux qui leur sont liés.

Les quatre derniers chapitres de l'ouvrage traitent des relations ville/ campagne, faisant notamment écho aux travaux pionniers de William Cronon sur Chicago et son hinterland. Ainsi, Stéphane Castonguay étudie sur un siècle l'évolution de l'agriculture dans la plaine de Montréal et démontre bien que, loin d'être tout simplement repoussée par le processus d'urbanisation, cette agriculture a su se réinventer et s'adapter au fil des décennies. Il démontre également avec rigueur que l'agriculture, dans la plaine de Montréal, obéit à des dynamiques différentes que dans le reste de la province. Les trois autres articles qui complètent cette section explorent également la complexité des rapports entre la métropole et sa périphérie, que ce soit dans le cadre de la chasse à courre pratiquée par le Montreal Hunt Club (Darcy Ingram), de la difficile intégration de la réserve de Kahnawake à l'espace métropolitain (Daniel Rueck) ou des répercussions dramatiques de la construction du canal de Beauharnois sur l'environnement rural de ce secteur (Louis-Raphaël Pelletier). Chacun de ces textes permet de mesurer la nature pour le moins artificielle de la frontière entre les mondes urbain et rural.

Bref, si cet ouvrage ne réinvente pas la roue sur le plan historiographique, s'inscrivant largement dans les grandes tendances en histoire environnementale nord-américaine, il permet de constater à quel point Montréal est un terrain fertile pour des études s'inscrivant dans ce champ de recherche et à quel point l'intérêt des historiens québécois pour l'histoire environnementale ne s'est pas démenti depuis les numéros spéciaux qu’y ont consacrés la Revue d'histoire de l'Amérique française et Globe en 2006.

HAROLD BÉRUBÉ Département d'histoire, Université de Sherbrooke 